\title{
Towards the development of a global Cn-continuous DIC procedure?
}

\author{
Wittevrongel L., Debruyne D., Lomov S.V. and Lava P. Department of Materials \\ Engineering, University of Leuven, Gebroeders de Smetstraat 1, 9000 Ghent BE
}

\begin{abstract}
In this work, a novel yet easy to use Global Digital Image Correlation algorithm is presented. The proposed method is developed to counter one of the important drawbacks of the most frequently adopted DIC techniques, namely the non-continuity of the strain field. In both the local subset method (no continuity) as in the widely known global Q4-DIC method ( $\mathrm{C}^{0}$-continuity) this problem arises. The proposed method is developed to obtain a $C^{1}$-continuous displacement field, and thus obtain a continuous strain field. In this way, strains can be derived directly from the algorithm without any post processing or filtering of the displacement, this in contrast to the previously mentioned DIC techniques. In these, the strain calculation is mostly done by using polynomial smoothing (e.g. strain window) of the displacement field. The extent of smoothing is controlled by user settings, making the measurement results significantly influenced by the user. The proposed algorithm uses well known principles both from the standard global DIC method as well as principles from finite element analysis to obtain a continuous strain field. Here an algorithm is presented using rather simple, specifically developed element shape functions, making the algorithm extremely easy to be implemented in a standard global approach.
\end{abstract}

Key words Strain continuity, Digital Image Correlation, Global Approach

\section{INTRODUCTION}

In most measurements performed by Digital Image Correlation, a $C^{1}$-continuous deformation field is expected. By expecting a continuous strain field, a measurement producing a smooth field can be considered as a more accurate measurement. In this context, it can be assumed that a digital image correlation algorithm producing a $C^{1}$-continuous deformation field is more accurate than current DIC approaches containing no or $C^{0}$-continuity. In the present article a special $C^{1}$-continuous DIC approach is developed to investigate whether the continuous method indeed outperforms the $C^{0}$ global approaches. The newly proposed algorithm is a global, using a triangular finite element mesh. The triangular elements within the mesh have a generic $p^{\text {th }}$ order description, so that elements can vary both in size as element order while remaining $\mathrm{C}^{1}$-continuity.

In a first stage, the general description for a global digital image correlation procedure is presented [1]. Next, some special shape functions are introduced to obtain the $C^{1}$-continuous elements.

\section{GLOBAL DIC}

In global DIC, the pixels are not individually but simultaneously tracked from the reference image $f(x)$ to the deformed image $g(\mathbf{x})$. The pixels are connected to each other using a finite element mesh. When defining the unknown displacement field for an element $\Omega$ within the mesh $\Delta$ as $\boldsymbol{d}$, the conservation of optical flow for each point $\mathbf{x}(\mathrm{x}, \mathrm{y})$ element of $\Omega$ writes:

$$
f(\boldsymbol{x}-\boldsymbol{d})=g(\boldsymbol{x})
$$

Similar as for the subset method specific cost functions are used to minimize the difference between $f(x-$ d) and $g(\boldsymbol{x})$. The correlation functions implemented are NSSD and ZNSSD but to maintain simplicity, the SSD is used to explain the method. The cost function $\epsilon$, based on the SSD and Eq 1, writes: 


$$
\in=\iint_{\Omega}\left[f(\boldsymbol{x}-\boldsymbol{d})-g\left(\boldsymbol{x}+\boldsymbol{d}^{\prime}\right)\right]^{2} d \boldsymbol{x}
$$

Where $\mathbf{d}^{\prime}$ is the previous solution used for the iterative calculation. When substituting the first order Taylor expansion of $f(x-\boldsymbol{d})$ into Eq. 2 the cost function becomes:

$$
\in=\iint_{\Omega}\left[\boldsymbol{d} \cdot \nabla f(\boldsymbol{x})+f(\boldsymbol{x})-g\left(\boldsymbol{x}+\boldsymbol{d}^{\prime}\right)\right]^{2} d \boldsymbol{x}
$$

Iterative calculation is critical as a Taylor expansion is used, and thus the calculated displacements are an approximation of the real displacement. Introducing a pre-described function for $\mathrm{d}$ on an arbitrary basis $\varphi$ can be written as:

$$
\boldsymbol{d}=\sum_{i, a} \varphi_{i} \cdot \delta_{i a} \cdot e_{a}
$$

where $i$ is the number of shape functions used, a are the system directions $(x, y)$ and $\delta_{i a}$ are the displacement parameters. The number of shape functions depends on the polynomial order of the element.

Minimising Eq. 3 using Eq. 4 with respect to $\delta_{i a}$, yields a linear equation:

with:

$$
\left[K_{i j a b}\right]^{e}\left[\delta_{i a}\right]^{e}=\left[F_{j b}\right]^{e}
$$

$$
\begin{gathered}
{\left[K_{i j a b}\right]^{e}=\iint_{\Omega} \varphi_{i} \cdot \nabla_{a} f(\boldsymbol{x}) \cdot \varphi_{j} \cdot \nabla_{b} f(\boldsymbol{x}) \cdot d \boldsymbol{x}} \\
{\left[F_{j b}\right]^{e}=\iint_{\Omega}\left[g\left(\boldsymbol{x}+\boldsymbol{d}^{\prime}\right)-f(\boldsymbol{x})\right] \cdot \varphi_{j} \cdot \nabla_{b} f(\boldsymbol{x}) \cdot d \boldsymbol{x}}
\end{gathered}
$$

where e is the element number, $\nabla_{a} f(\boldsymbol{x})$ is the derivative of the function with respect to $\mathrm{a}$ and $\mathrm{i}$ and $\mathrm{j}$ denote the number of shape functions, depending on the element order. To include the connectivity of the mesh, all element equations are assembled to one linear "system equation".

$$
[K]^{s}[\delta]^{s}=[F]^{s}
$$

\section{CO SHAPE FUNCTIONS}

The first choice for the shape functions can be Legendre functions. These were first introduced in p-DIC [2]. The hierarchical functions are based on Legendre polynomials, the same functions as often used in adaptive p-elements [3]. The shape functions can be obtained using a combination of function $\mathrm{P}_{\mathrm{p}}$ :

$$
P_{p}(x)=\frac{1}{(p-2) ! 2^{p-2}} \frac{d^{p-2}}{d(x)^{p-2}}\left[\left(1-x^{2}\right)^{p-1}\right]
$$

These functions are assigned to nodes (vertex modes), edges (side modes) and faces (internal modes) of the elements as shown in Figure 1. 


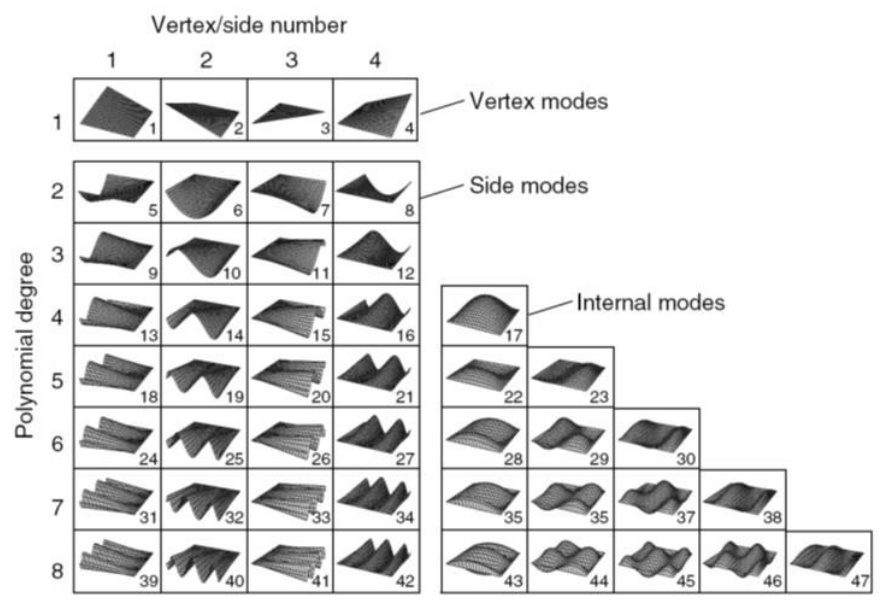

Fig 1: Hierarchical Shape functions [3]

In its first order configuration it represents the same functions as the Lagrange description, as used in [1]. The difference occurs when higher order descriptions are used. To obtain a higher order description, extra displacement functions can be added to the displacement without altering the first order shape functions. This characteristic lead to an adaptive global DIC approach named p-DIC. Using the hierarchical shape functions, element orders are not limited and can be increased during correlation. Increasing element orders is also known as a p-refinement scheme, hence the name p-DIC. Even further, automatic refinement procedures where implemented so the algorithm became self adaptive to fit the measured displacement field.

\section{C1 SHAPE FUNCTIONS}

To implement $C^{1}$-continuity, some alternative shape functions $\varphi_{i}$ should be used. Traditional shape functions such as Lagrange or Legendre shape functions will only introduce $C^{0}$ continuity. As shown in [2], the use of higher order elements in a global DIC approach has a positive influence on the correlation procedure. For this reason, not simply fixed $C^{1}$-continuous functions are used but a set of generic $\mathrm{p}^{\text {th }}$ order functions is introduced. In this way a similar $\mathrm{p}$-refinement scheme is possible but $\mathrm{C}^{1}$-continuity is included.

Element Geometry As a starting base, Argyris elements are used to obtain $C^{1}$ continuous shape functions. The Argyris element, shown in Fig. 2, contains 21 degrees of freedom (DOF) representing a $5^{\text {th }}$ order element. The starting order is 5 , since no $C^{1}$-functions exist for $p<5$.

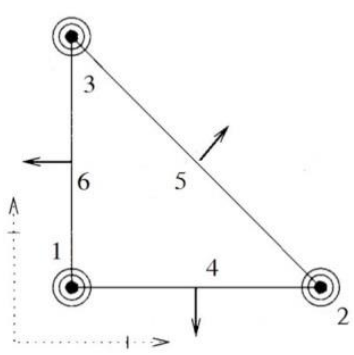

Fig 2: The Argyris element, containing 21 DOF

It has so-called "Argyris DOF" (1-3) at the vertices and "Hermite DOF" (4-6) at the center of the edges. The degrees of freedom for the different points are:

Argyris point $\left(\mathrm{N}_{\mathrm{A}}\right)$ has DOF:

$$
d, \frac{\delta d}{\delta x}, \frac{\delta d}{\delta y}, \frac{\delta^{2} d}{\delta^{2} x}, \frac{\delta^{2} d}{\delta^{2} y} \text { and } \frac{\delta^{2} d}{\delta x y}
$$


Hermite point $\left(\mathrm{N}_{\mathrm{H}}\right)$ had DOF:

$$
\frac{\delta d}{\delta n}
$$

where $\mathrm{n}$ is the normal direction on the considered edge. It is shown that using the standard DOF (Fig. 1) the solution is both unisolvent and conforming to the space $\mathrm{H}^{2}$, and thus containing continuous differentiation across boundaries. To obtain higher order elements, more DOF has to be introduced to the element in order to represent a higher polynomial order. It is shown that in the general case one needs $p-5$ Lagrange DOF (only using $d$ as DOF) and $p-4$ Hermite DOF per edge in order to satisfy the requirements of the $\mathrm{H}^{2}$ space .

Lagrange point $\left(\mathrm{N}_{\mathrm{L}}\right)$ has DOF:

$d$

In total, resulting in 6p-9 DOF for a $\mathrm{p}^{\text {th }}$ order element. The number of degrees of freedom needed to describe a $\mathrm{p}^{\text {th }}$ order polynomial writes:

$$
N_{p}=\frac{(p+1)(p+2)}{2}
$$

As $N_{p}>6 p-9$, the element is completed with internal Lagrange points to describe a full $p^{\text {th }}$ order polynomial so that $\mathrm{N}_{\mathrm{p}}=\mathrm{N}_{\mathrm{L}}+\mathrm{N}_{\mathrm{H}}+\mathrm{N}_{\mathrm{A}}+\mathrm{N}_{\mathrm{L} \text { internal. }}$

$$
N_{L \text { internal }}=\frac{p^{2}-9 p+20}{2}
$$

Using the above, the sixth and seventh order elements are shown in Fig 3.
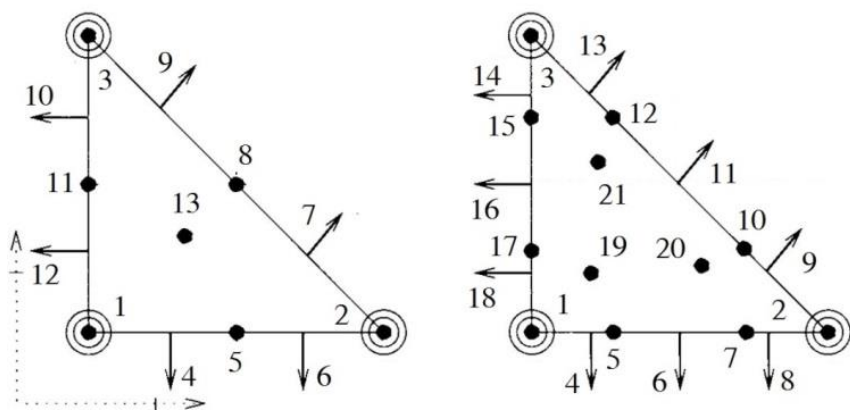

Fig 3: Sixth and seventh order element.

Shape Functions Using the $p^{\text {th }}$ order description for the value $\psi$, representing horizontal or vertical displacement, can be written as:

$$
\psi=\sum_{n=1}^{N_{p}} a_{n} x^{k_{n}} y^{l_{n}}=\sum_{n=1}^{N_{p}} a_{n} g_{n}
$$

Where $k_{n}$ and $I_{n}$ represent the powers for $x$ and $y$ based on Pascals Triangle and $a_{n}$ represents the sought polynomial coefficients. Function $g_{n}$ is part of the basis $\beta=\left\{g_{1}, g_{2}, g_{3}, \ldots, g_{N p}\right\}$, defined by the polynomial order $p$ of the element. Matrix $[\psi]$ can be defined as the matrix containing the degrees of freedom for all nodes of a certain element. The degrees of freedom for each node are dependent on its type, as mentioned above. 


$$
[\psi]=\left[D O F_{1}, D O F_{2}, \ldots, D O F_{n}\right]
$$

Using matrix $[\psi]$, following relationship can be established:

$$
[\psi]=[L][a]
$$

where matrix $[a]$ represents the polynomial coefficients from Eq. 11 and $[L]$ represents the generalised Vandermonde matrix $L=\left\{L_{i}\left(g_{i}\right)\right\}_{i, j=1}^{N_{p}}$ using the basis $\beta$. In matrix $[L] \mathrm{L}_{i}$ depends on DOF number $\mathrm{i}$, linked to the type of each node.

Using the basic condition for a set of shape functions:

$$
\varphi_{i}\left(x_{j}, y_{j}\right)=\left\{\begin{array}{l}
1 \text { if } i=j \\
0 \text { if } i \neq j
\end{array}\right.
$$

$\left[\psi^{i}\right]$ Can then be defined as:

$$
\left[\psi^{i}\right]_{j}=\left\{\begin{array}{l}
1 \text { if } i=j \\
0 \text { if } i \neq j
\end{array}\right.
$$

Shape function $\varphi_{i}(x, y)$ can then be found by:

$$
\begin{aligned}
\varphi_{i}(x, y) & =[a][g] \\
& =[L]^{-1}\left[\psi^{i}\right][g]
\end{aligned}
$$

Where

$$
\begin{array}{rlc}
{[g]} & = & {\left[g_{1}, g_{2}, \ldots, g_{N p}\right]} \\
& = & {\left[x^{k_{0}} y^{l_{0}}, x^{k_{1}} y^{l_{1}}, \ldots, x^{k_{N p}} y^{l_{N p}}\right]}
\end{array}
$$

The shape functions above can be implemented in the standard global DIC approach (Eq. 6 and 7) to obtain a $C^{1}$-continuous strain field. In the following, the newly proposed method will be compared to the hierarchical approach and the traditional subset method to determine whether a $\mathrm{C}^{1}$-continuous method indeed outperforms the non-continuous methods.

\section{COMPARISON}

To compare the different DIC approaches, the resolutions and corresponding spatial resolutions are plotted in one graph, as the combination of these two quantities indicate the performance of the methods. How these values where determined, the reader is referred to [2] and [4]. As can be predicted, both values are inversely related. Achieving a lower spatial resolution leads to an increase of the measurand resolution. For comparison, the in-house developed platforms "MatchID 2D" [5] and "AdaptID" are used. "MatchID 2D" implements the local subset method, while "AdaptID" implements both the automatic refined global higher order approach and the $C^{1}$-approach. All three methods (subset, $C^{0}$ and the newly proposed method $C^{1}$ ) use same libraries for interpolation and mathematical operations, leading to a more profound comparison. The influence of filters, interpolation and matrix calculations are ruled out in this way.

\begin{tabular}{llll}
\hline & Subset & $\mathbf{C}^{0}$ & $\mathbf{C}^{1}$ \\
\hline Principle & Local & Global & Global \\
Element & Square & Curved Quad & Triangle \\
Order & $0 \rightarrow 2$ & $1 \rightarrow p$ & $5 \rightarrow p$ \\
Continuity & Non & Displacement & Strain \\
\hline
\end{tabular}


The results for the validation of displacement and strain resolution versus spatial resolution are presented in Figures 4 and 5. The data indicates that the global approaches, $C^{0}$ and $C^{1}$, are more robust to noise then the local subset method. The more noise robustness of a Q4-mesh compared to the subset method using the same element size has already been proven in [6]. Note that this study only covers the noise robustness and not the link to the spatial resolutions.

When $C^{1}$ and $C^{0}$ are compared to each other, it is seen that the introduction of $C^{1}$-continuity does not lead to an extra gain in resolution. Even more, the constraint of $\mathrm{C}^{1}$-continuity increases in spatial resolution as it limits the flexibility of the element. In fact, introducing continuity did improve the resolution but was not able to overcome the loss in spatial resolution. It can be concluded that expecting a continuous strain field and thus using a $\mathrm{C}^{1}$-continuous algorithm does not necessarily lead to better results. The use of the hierarchical higher order approach results in better resolutions. Combined with the less user dependent results by the automatic refinement procedure, $\mathrm{C}^{0}$ is the more favorable DIC algorithm. It is noted though that the results of the newly proposed method where quite competitive and thus, when explicitly a $C^{1}$ continuous filed is requested, e.g. with beam kinematics, the c-DIC approach is a valuable alternative towards the $\mathrm{C}^{0}$ method.

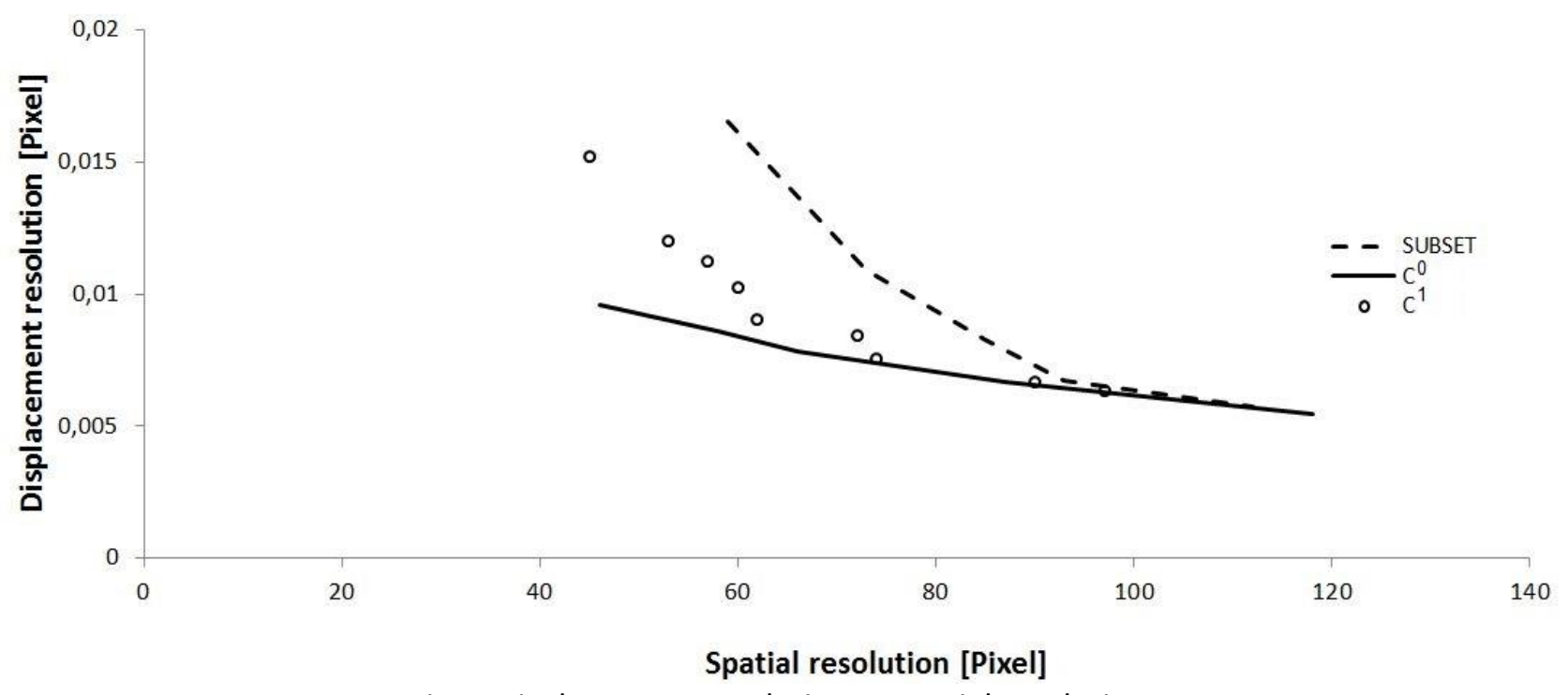

Fig 4: Displacement resolution vs spatial resolution.

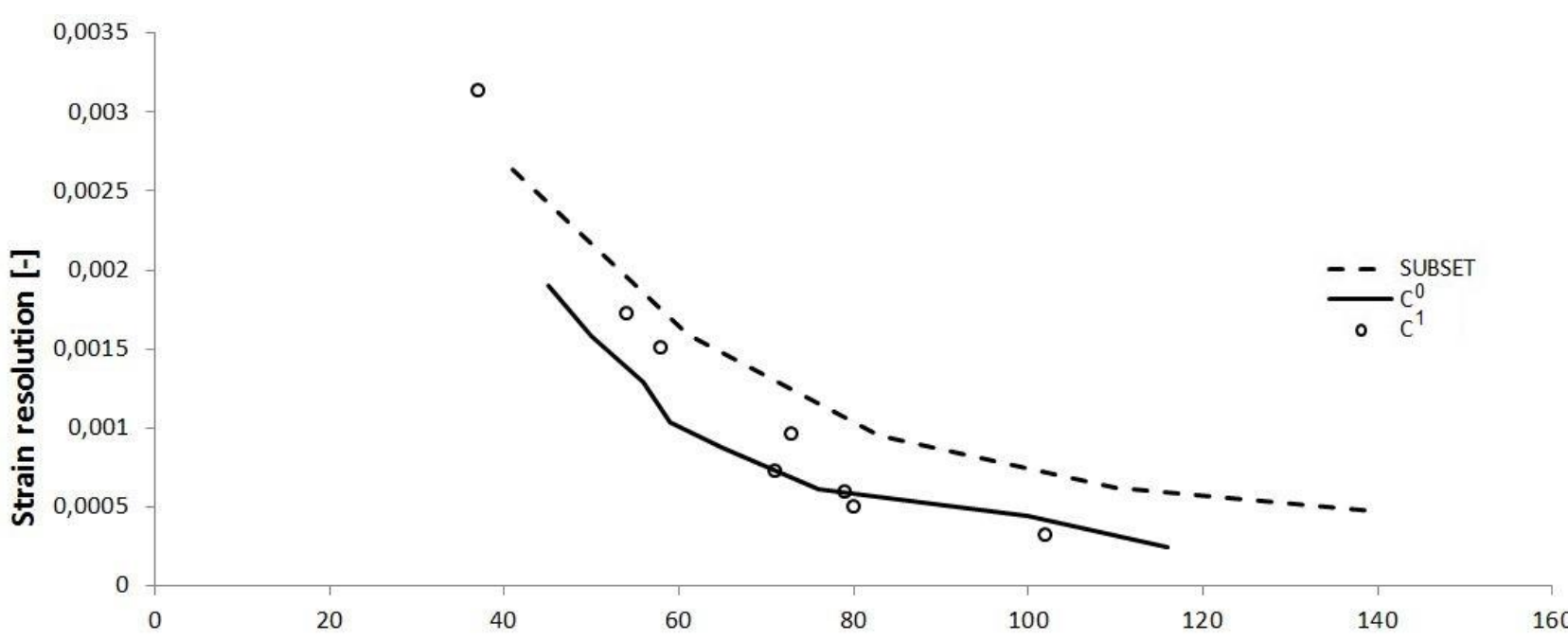

Spatial resolution [Pixel]

Fig 5: Strain resolution vs spatial resolution. 


\section{CONCLUSION}

In this article a novel $\mathrm{C}^{1}$-contiuous global DIC algorithm is presented. It is based on a standard global DIC description but uses specific developed $C^{1}$-contiuous elements with a generic $p^{\text {th }}$ order description. The generic description allows that elements can vary in both size as order while remaining $C^{1}$-contiuous. It is validated whether an algorithm containing continuous strains can be considered as more accurate than some alternative algorithms containing no continuity. This statement is derived from the intuitive feeling that a smooth strain field is more accurate than a non-smooth field. This assumption is investigated with the special developed algorithm implemented in the AdaptID platform. The newly proposed approach is compared to the traditional subset method (MatchID) and an adaptive higher order global approach containing $\mathrm{C}^{0}$-continuity, also implemented in the AdaptID platform. Both platforms use same libraries for interpolation and mathematical operations, leading to a more profound comparison. It is concluded that the $p^{\text {th }}$ order global $C^{1}$-contiuous DIC approach is competitive against the other approaches, but does not outperform the methods. Using the global approach increases the more robustness to noise, but the constraint of $C^{1}$-continuity limits the elements deformation flexibility. With this investigation it is made clear that including $C^{1}$-continuity does not provide as much benefits as could be instinctively expected. Due to the automatic refinement and great performance, the adaptive higher order global approach (AdaptID $C^{0}$ ) is considered as more valuable. Mark that because the results where competitive, AdaptID $C^{1}$ can be a valuable alternative when $C^{1}$-continuity is explicitly requested.

\section{REFERENCES}

[1] Besnard G. et al, "Finite-Element" displacement fields analysis from digital images: application to portevin-la chatelier bands, Experimental Mechanics volume 46 issue 6 pp789-803 (2006)

[2] Wittevrongel L. et al, A self adaptive global digital image correlation algorithm, Experimental Mechanics DOI: 10.1007/s11340-014-9946-3 (2014)

[3] Szabo B. et al, The p-version of the finite element method, Encyclopedia of Computational Mechanics (2004)

[4] Bornet M. et al, Assessment of digital image correlation measurement errors: methodology and results, Experimental Mechanics volume 49 issue 3 pp353-370 (2008)

[5] MatchID 2D, http://www.matchidmbc.be/

[6] Hild F. et al, Comparison of local and global approaches to digital image correlation, Experimental Mechanics volume 52 issue 9 pp1503-1519 (2012) 